\title{
Expert Review of Neurotherapeutics
}

\section{Protective roles of intestinal microbiota derived short chain fatty acids in Alzheimer's disease-type beta-amyloid neuropathological mechanisms}

Lap Ho, Kenjiro Ono, Mayumi Tsuji, Paolo Mazzola, Risham Singh \& Giulio M. Pasinetti

To cite this article: Lap Ho, Kenjiro Ono, Mayumi Tsuji, Paolo Mazzola, Risham Singh \& Giulio M. Pasinetti (2018) Protective roles of intestinal microbiota derived short chain fatty acids in Alzheimer's disease-type beta-amyloid neuropathological mechanisms, Expert Review of Neurotherapeutics, 18:1, 83-90, DOI: $10.1080 / 14737175.2018 .1400909$

To link to this article: https://doi.org/10.1080/14737175.2018.1400909

Accepted author version posted online: 02

Nov 2017.

Published online: 14 Nov 2017.

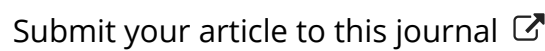

山 Article views: 137

Q View related articles ¿

View Crossmark data 


\title{
Protective roles of intestinal microbiota derived short chain fatty acids in Alzheimer's disease-type beta-amyloid neuropathological mechanisms
}

\author{
Lap Ho ${ }^{a *}$, Kenjiro Ono ${ }^{b *}$, Mayumi Tsujic, Paolo Mazzola $\mathbb{1}^{\mathrm{d}}$, Risham Singh and Giulio M. Pasinettia

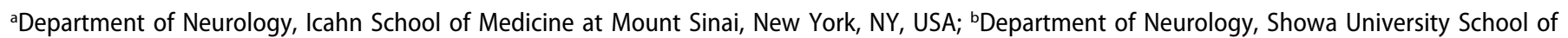 \\ Medicine, Tokyo, Japan; 'Department of Pharmacology, Showa University School of Medicine, Tokyo, Japan; ${ }^{d}$ Department of Medicine, Center for \\ Neuroscience, University of Milano-Bicocca, Monza, Italy
}

ABSTRACT

Background: Dietary fibers are metabolized by gastrointestinal (GI) bacteria into short-chain fatty acids (SCFAs). We investigated the potential role of these SCFAs in $\beta$-amyloid $(A \beta)$ mediated pathological processes that play key roles in Alzheimer's disease (AD) pathogenesis.

Research design and methods: Multiple complementary assays were used to investigate individual SCFAs for their dose-responsive effects in interfering with the assembly of $A \beta B 1-40$ and $A \beta 1-42$ peptides into soluble neurotoxic $A \beta$ aggregates.

Results: We found that several select SCFAs are capable of potently inhibiting $A \beta$ aggregations, in vitro. Conclusion: Our studies support the hypothesis that intestinal microbiota may help protect against $A D$, in part, by supporting the generation of select SCFAs, which interfere with the formation of toxic soluble $A \beta$ aggregates.
ARTICLE HISTORY

Received 18 August 2017 Accepted 1 November 2017

KEYWORDS

Alzheimer's disease;

beta-amyloid $(A \beta)$; fibrils; protein misfolding; microbial; microbiome; microbiota;

neurodegeneration

\section{Introduction}

The term intestinal microbiota refers to the tens of trillions of commensal, symbiotic, and pathogenic microorganisms, including bacteria, fungi, and archaea, which live in our intestine. There is a growing interest in the potential contributions of intestinal microbiota, particularly among the intestinal bacterial population, in human health and/or disease [1]. There is a tremendous diversity among individuals' intestinal microbiota with respect to the composition of specific bacterial species and the density (number) of bacteria that are present for each of these bacterial species. Indeed, such interpersonal differences in intestinal bacteria composition have been associated with the presence or absence of an increasing number of health issues, including metabolic syndrome, obesity, immunological diseases, cardiovascular diseases, as well as neurodegenerative disorders such as Alzheimer's disease (AD) and Parkinson's disease (PD) [1-4].

Gastrointestinal (Gl) bacteria may affect host functions by interfering with potential pathogens, improving barrier function, immunomodulation, and/or production of neurotransmitters [5]. Gl bacteria may also affect host functions through diet-based microbial influences, by metabolizing dietary compounds into readily absorbable, biologically available, bioactive forms that are responsible for modulation of select biological processes [6]. Accumulating evidence has demonstrated that such diet-based microbial influences may help promote resilience against diverse medical conditions, including neurological disorders such as $A D$, by modulation of metabolic and immunologic responses and/or other disease-specific pathological mechanisms [7-10]. An example of such diet-based microbial influence on neurodegenerative disorders is the critical role of gut microbiota in the bioactivity of dietary polyphenols. It has been estimated that about $90 \%$ of the dietary polyphenols are not absorbed by the small intestine and are accumulated in the colon where they are subjected to metabolism by GI microbial into phenolic acids, which are then readily absorbed $[11,12]$. Recent evidence revealed that some of these biologically available phenolic acids such as caffeic acid and ferulic acid [13-16] are bioactive in inhibiting the generation of beta-amyloid $(A \beta)$ peptides, a key pathogenic feature of $A D$ [8], as well as in suppressing the elevated oxidative stress and inflammatory responses that are observed in $A D$ as well as in other neurodegenerative disorders $[9,10]$. Moreover, our recent evidence revealed that other biologically available, GI microbiota-derived phenolic acids, such as 3-hydroxybenzoic acid and 3-(3'-hydroxyphenyl)propionic acid, are bioactive in interfering with the misfolding of $A \beta$ peptides into neurotoxic $A \beta$ aggregates that play key roles in AD pathogenesis [17]. Collectively, this evidence suggests that the GI microbiota may help attenuate the development and/or progression of $A D$ through the generation of microbiota-derived phenolic compounds that mechanistically target diverse pathogenic mechanisms underlying AD.

Another example of diet-based microbial influence on $A D$ is the involvement of $\mathrm{Gl}$ microbials in the metabolism of 
dietary fibers. Dietary fibers are carbohydrate polymers, which cannot be hydrolyzed by the endogenous enzymes in the upper gut. Instead, dietary fibers are metabolized by the microbiota in the colon into short-chain fatty acids (SCFAs) with five carbons or less, including valeric acid and isovaleric acid (C5), isobutyric acid and butyric acid (C4), propionic acid (C3), acetic acid (C2), and formic acid (C1) $[18,19]$. It has been hypothesized that SCFAs generated by $\mathrm{Gl}$ bacterial metabolism of dietary fibers may attenuate $A D$ by serving as substrates for energy metabolism [7], and providing an alternative energy source to rectify brain hypometabolism that contributes to neuronal dysfunctions in $A D$ and other neurodegenerative conditions [20]. Recent evidence suggests that select SCFAs may also help modulate maturation and function of microglia in the brain [21], implicating the potential benefits of $\mathrm{Gl}$ bacteria-derived SCFAs in modulating neuro-inflammatory processes that play important roles across diverse neurodegenerative disorders, including AD. More recently, it was shown that GI bacterial-mediated generation of butyric acid, an SCFA from dietary fibers, may provide therapeutic benefits for $A D$ through epigenetic mechanisms of action by inhibiting histone deacetylase and normalizing aberrant histone acetylation in $A D$ [22]. However, while neurotoxic $A \beta$ aggregates play a central role in $A D$ onset and progression, it is currently unknown whether Gl bacteria-derived SCFAs may modulate protein misfolding. Therefore, we hereby investigate whether GI bacteria-derived SCFAs may help modulate the self-assembly of $A \beta$ peptide, in vitro, using established assays. Outcomes from this study provide critical information for developing probiotics to help prevent and/or treat AD.

\section{Methods}

\subsection{Chemicals and solvents}

Chemicals were obtained from Sigma and, unless otherwise stated, were of the highest purity available. Solvents were High-Performance Liquid Chromatography (HPLC) grade and were obtained from Fisher. Water was double-distilled and deionized using a Milli-Q system (Millipore Corp., Bedford, MA).

\subsection{Peptides and proteins}

Monomeric $A \beta 1-40$ and $A \beta 1-42$ peptides were synthesized, purified, and characterized as described previously [23]. Purified peptides were stored as lyophilizates at $-20^{\circ} \mathrm{C}$. A stock solution of glutathione S-transferase (GST; SigmaAldrich) was prepared by dissolving the lyophilizate to a concentration of $250 \mu \mathrm{M}$ in $60 \mathrm{mM} \mathrm{NaOH}$. Prior to use, aliquots were diluted 10 -fold into $10 \mathrm{mM}$ sodium phosphate, $\mathrm{pH} 7.4$.

\subsection{Preparation of $A \beta$ solutions}

Aggregate-free $A \beta 1-40$ or $A \beta 1-42$ solutions were prepared as described previously [24]. To prepare $A \beta, 200 \mu$ l of a
$2 \mathrm{mg} / \mathrm{ml}$ peptide solution in dimethyl sulfoxide was sonicated for $1 \mathrm{~min}$ using a bath sonicator (Branson Ultrasonics, Danbury, CT) and then centrifuged for $10 \mathrm{~min}$ at $16,000 \times \mathrm{g}$. The resulting supernate was fractionated on a Superdex 75 HR column using $10 \mathrm{mM}$ phosphate buffer, $\mathrm{pH} 7.4$, at a flow rate of $0.5 \mathrm{ml} / \mathrm{min}$. The middle of the low-molecularweight (LMW) peak was collected around $25 \mathrm{~min}$ and used immediately. A 10- $\mu$ l aliquot was taken for amino acid analysis to determine quantitatively the peptide concentration in each preparation. Typically, the concentrations of $A \beta 1-40$ and $A \beta 1-42$ were $30-40$ and $10-20 \mu \mathrm{M}$, respectively.

\subsection{Peptide aggregation}

Aggregation of $A \beta 1-40$ (or $A \beta 1-42$ ) peptide was conducted essentially as described previously [25]. In brief, $A \beta$ solutions $(0.5-\mathrm{ml}$ aliquots) were placed in $1-\mathrm{ml}$ microcentrifuge tubes. Test compounds (SCFAs) were dissolved in ethanol to a final stock concentration of $2.5 \mathrm{mM}$. Peptide aggregation was conducted in $10 \mathrm{mM}$ phosphate, $\mathrm{pH} 7.4$, with $5 \mu \mathrm{M} \mathrm{A \beta 1-40}$ (or $A \beta 1-42$ ) peptide in the presence of either vehicle or individual SCFA a final $5 \mu \mathrm{M}$ or $20 \mu \mathrm{M}$ concentration, for a final SCFA:A $\beta$ molar ratio of $1: 1$ or $1: 4$. The tubes were incubated at $37^{\circ} \mathrm{C}$ for $0-10$ days without agitation.

\subsection{Photoinduced cross-linking of unmodified proteins}

$A \beta$ peptide oligomer frequency distributions were assessed using the photoinduced cross-linking of unmodified proteins (PICUP) protocol as described previously [25]. Briefly, $1 \mu \mathrm{l}$ of $1 \mathrm{mM}$ tris(2,2'-bipyridyl)dichlororuthenium(II) (Ru (bpy)) and $1 \mu \mathrm{l}$ of $20 \mathrm{mM}$ ammonium persulfate were added to $18 \mu \mathrm{l}$ of freshly prepared protein solution. We noted that addition of SCFA did not lead to observable change in the $\mathrm{pH}$ of reaction mixture. The mixture was irradiated for $1 \mathrm{~s}$ with visible light, and then the reaction was quenched with $10 \mu \mathrm{l}$ of Tricine sample buffer (Invitrogen) containing 5\% (v/v) $\beta$-mercaptoethanol. An aliquot $(20 \mu \mathrm{l})$ of each cross-linked sample was electrophoresed on a $10-20 \%$ gradient Tricine gel and visualized by silver staining (SilverXpress, Invitrogen). Non-cross-linked samples were used as controls in each experiment. To produce intensity profiles and calculate the relative amounts of each oligomer type, Densitometry was performed, and OneDscan software (v. 2.2.2; BD Biosciences Bioimaging) was used to determine peak areas of baseline corrected data.

\subsection{Thioflavin T spectroscopic assay}

The thioflavin T (ThT) assay was conducted essentially as described previously [25]. Ten microliter of sample was added to $190 \mu \mathrm{l}$ of ThT dissolved in $10 \mathrm{mM}$ phosphate buffer, $\mathrm{pH} 7.4$, and then the mixture was vortexed briefly. ThT fluorescence was determined three times at intervals of $10 \mathrm{~s}$ using an Hitachi F-4500 fluorometer. Excitation and emission wavelengths were 450 and $482 \mathrm{~nm}$, respectively. Sample fluorescence was determined by averaging the three readings and subtracting the fluorescence of a ThT blank. 


\subsection{Electron microscopy}

To study protofibril formation and the effects of SCFAs on it, $A \beta$ was incubated according to the aggregation protocol above. After incubation at $37^{\circ} \mathrm{C}$ for 7 days in $10 \mathrm{mM}$ sodium phosphate, $\mathrm{pH} 7.4$, Electron microscopy (EM) was used to determine the morphologies of $A \beta 1-40$ or $A \beta 1-42$ assemblies as described previously [25]. Briefly, a 10- $\mu$ l aliquot of each sample was spotted onto a glow-discharged, carbon-coated Formvar grid (Electron Microscopy Sciences, Hatfield, PA) and incubated for $20 \mathrm{~min}$. The droplet then was displaced with an equal volume of $2.5 \%(\mathrm{v} / \mathrm{v})$ glutaraldehyde in water and incubated for an additional 5 min. Finally, the peptide was stained with $8 \mu \mathrm{l}$ of $1 \%(\mathrm{v} / \mathrm{v})$ filtered $(0.2 \mu \mathrm{M})$ uranyl acetate in water (Electron Microscopy Sciences). This solution was wicked off, and then the grid was air dried. Samples were examined using a JEOL CX100 transmission electron microscopy.

\section{Results}

\subsection{Effects of SCFAs on $A \beta$ protein-protein interactions}

The self-assembly of $A \beta$ peptides into neurotoxic soluble $A \beta$ aggregates is one of the key neuropathological processes underlying AD. We used the PICUP assay to monitor the effect that individual SCFAs have in interfering with initial protein- protein interactions necessary for the assembly of $A \beta 1-40$ or $A \beta 1-42$ peptides into neurotoxic aggregates in the presence or absence of individual SCFAs at a SCFA:A $\beta$ molar ratio of 1:1 or 1:4. Six SCFAs were tested using the PICUP assay: valeric acid, isovaleric acid, butyric acid, isobutyric acid, propionic acid, and acetic acid. Monomeric A $\beta 1-40$ or $A \beta 1-42$ peptides and cross-linked multimeric $A \beta$ forms were resolved by sodium dodecyl sulfate polyacrylamide gel electrophoresis (SDS-PAGE) and visualized by staining.

In the absence of cross-linking, only $A \beta 1-40$ monomers were observed (Figure 1(a,b), lane 1). As we have previously reported [26], cross-linking of $A \beta 1-40$ in the absence of SCFAs leads to the formation of $A \beta 1-40$ dimer and trimer forms (Figure $1(a, b)$, lane 2 , indicated by black arrow). We observed that valeric acid potently interferes with initial $A \beta 1-40$ proteinprotein interactions. In particular, the addition of valeric acid at a SCFA:A $\beta$ molar ratio of 1:1 (Figure $1(a, b)$, lane 13 ) completely inhibited the formation of the trimeric $A \beta 1-40$ form and partially inhibited the formation of the dimer $A \beta 1-40$ form (compare lane 13 vs. lane 2). The addition of an increasing molar ratio of valeric acid with a SCFA:A $\beta$ molar ratio of $4: 1$ (Figure $1(a, b)$, lane 14$)$ completely blocked the formation of into dimeric or trimeric $A \beta 1-40$ forms (compare lane 14 vs. lane 2). Both butyric acid and propionic acid also interfered with $A \beta 1-40$ oligomerization but to a lesser extent in a
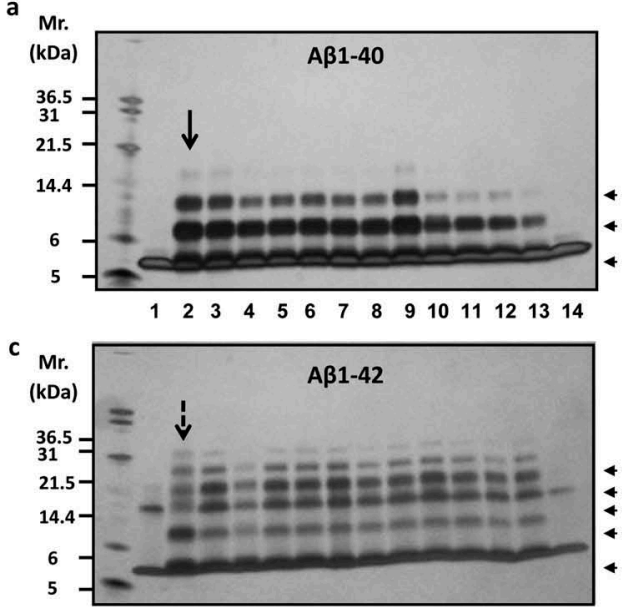

$\begin{array}{lllllllllllllll}1 & 2 & 3 & 4 & 5 & 6 & 7 & 8 & 9 & 10 & 11 & 12 & 13 & 14\end{array}$

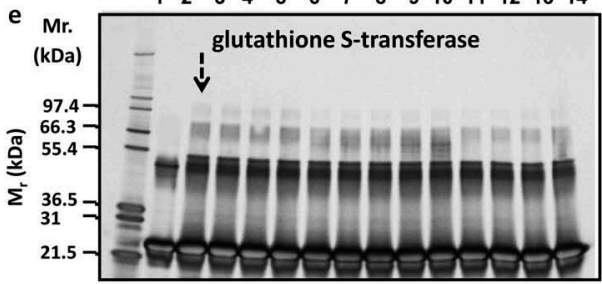

$\begin{array}{llllllllllllll}1 & 2 & 3 & 4 & 5 & 6 & 7 & 8 & 9 & 10 & 11 & 12 & 13 & 14\end{array}$
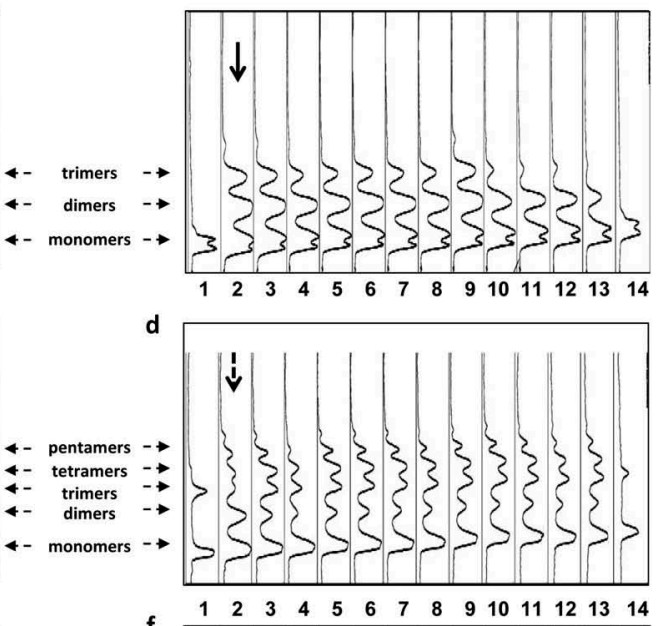

f

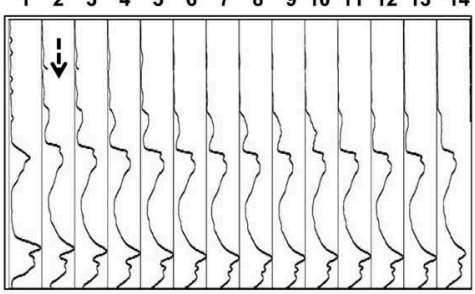

$\begin{array}{llllllllllllll}1 & 2 & 3 & 4 & 5 & 6 & 7 & 8 & 9 & 10 & 11 & 12 & 13 & 14\end{array}$

Figure 1. Select SCFAs potently interfere with protein-protein interactions among A? peptides. $(a, c$, e) Monomeric, dimeric and higher-ordered cross-linked multimeric A?1-40 (a), A? 1-42 (c) or GST (e) aggregates were visualized by silver staining of the gel. Shown are representative assays from three independent studies. (b, d, f) Densitometry intensity profiles for A?1-40 (b), A?1-21 (d) and GST (f). In (a-f, lane 1) A?1-40 (a,b), A?1-42 (c,d) or GST (e,f) alone without cross-linking. In (a-f, lanes 2-14) A?1-40 (a,b), A?1-42 (c,d) or GST (e,f) with cross-linking in the presence of vehicle (lane 2) or in the presence of individual SCFSs as follow: isobutyric acid at a SCFA:A? (or GST) molar ratio of 1:1 (lane 3) or 4:1 (lane 4), isovaleric acid at a SCFA:A? molar ratio of 1:1 (lane 5) or 4:1 (lane 6), acetic acid at a SCFA:A? (or GST) molar ratio of 1:1 (lane 7) or 4:1 (lane 8), propionic acid at a SCFA:A? (or GST) molar ratio of 1:1 (lane 9) or 4:1 (lane 10), butyric acid at a SCFA:A? (or GST) molar ratio of 1:1 (lane 11) or 4:1 (lane 12), valeric acid at a SCFA:A? (or GST) molar ratio of 1:1 (lane 13) or 4:1 (lane 14). In (a-d), horizontal arrows indicate monomers, dimers, trimers, tetramers and pentamers. In (a-f), vertical arrows indicate positive control studies in which A?1-40 (a-b), A?1-42 (c-d) or GST (e-f) were incubated in the absence of SCFA. 
comparison to valeric acid. In particular, butyric acid, at a SCFA:A $\beta$ molar ratio of $1: 1$ (Figure $1(a, b)$, lane 11) or $4: 1$ SCFA:A $\beta$ (Figure $1(a, b)$, lane 12), almost completely inhibited the aggregation of $A \beta 1-40$ monomers into $A \beta 1-40$ trimers, but was not effective in modulating the formation of $A \beta 1-40$ dimeric forms (compare lanes 11 and 12 vs. lane 2). Propionic acid, at a SCFA:A $\beta$ ratio of $4: 1$ (Figure $1(a, b)$, lane $10)$, almost completely inhibited the generation of $A \beta 1-40$ trimers (compare lane 10 vs. lane 2). However, $A \beta 1-40$ aggregation was not affected by the addition of a lower molar ratio of propionic acid, at a 1:1 SCFA:A $\beta$ molar ratio (Figure $1(a, b)$, lane 9) (compare lane 9 vs. lane 2). The remaining three SCFAs monitored (isobutyric acid, isovaleric acid, and acetic acid) had no observable impact on the conversion of monomeric $A \beta 1-40$ peptide (Figure $1(a, b)$, lanes $3-8$ ) into higher-ordered multimeric $A \beta 1-40$ forms in our PICUP assay (compare lanes $3-8$ vs. lane 2).

In the absence of cross-linking, monomeric $A \beta 1-42$ shows up in a trimeric form under SDS-PAGE (Figure 1(c,d), lane 1). The $A \beta 1-42$ trimer band has been shown to be an SDS-induced artifact $[26,27]$. As we have previously reported [26], cross-linking of $A \beta 1-42$ in the absence of SCFAs leads to the formation of $A \beta 1-42$ oligomers of orders 2-6 (Figure 1(c,d), lane 2, indicated by black arrow). We observed that the addition of valeric acid, at a SCFA:A $\beta$ molar ratio of $4: 1$ (Figure $1(c, d)$, lane 14 ), completely inhibited the formation of all $A \beta 1-42$ oligomers (compare lane 14 vs. lane 2). However, $A \beta 1-42$ aggregation was not affected by the addition of a lower molar ratio of valeric acid, at a 1:1 SCFA:A $\beta$ molar ratio (Figure $1(c, d)$, compare lane 13 vs. 2 ). The conversion of $A \beta 1-42$ monomers into higher-order multimeric forms was unaffected by the addition of butyric acid, propionic acid, isobutyric acid, isovaleric acid, and acetic acid at either a SCFA:A $\beta$ molar ratio of $1: 1$ or $4: 1$ (Figure 1(c,d), compare lanes 2-12 vs. lane 2).

In parallel, in the control PICUP study, which used GST as a positive control for the cross-linking chemistry as we have used in the past [25], we observed that incubation of GST in the absence of SCFAs leads to the formation of higher molecular weight GST aggregate forms (Figure 1(e,f), lane 2, indicated by a black arrow). We observed no alterations in GST cross-linking in the presence of SCFAs at a SCFA:GST molar ratio of 1:1 (Figure $1(\mathrm{e}, \mathrm{f})$ ), compare lanes $3,5,7,8,9,11,13$ vs. lane 2 ) or 4:1 (Figure $1(\mathrm{e}, \mathrm{f})$, compare lanes $4,6,8,10,12,14$ vs. lane 2). We therefore concluded that valeric acid, butyric acid, and propionic acid inhibited $A \beta 1-40$ and/or $A \beta 1-42$ aggregation.

\subsection{Effects of SCFAs on $A \beta$ fibril formation}

Based on the evidence from our PICUP assay, we continued by assessing valeric acid and butyric acid for their potential effects on the assembly of monomeric $A \beta$ peptides into $A \beta$ fibrils using the ThT spectroscopic assay to monitor for temporal changes in $\beta$-sheet contents of $A \beta 1-40$ and $A \beta 1-42$, which were incubated in the absence of SCFA (SCFS:A $\beta$ molar ratio of $0: 1$ ) or in the presence of individual SCFAs at a
SCFA:A $\beta$ molar ratio of $1: 1$ or $4: 1$. We note that ThT fluorescence is not a direct measure of fibril content. However, since $\beta$-sheet formation correlates with fibril formation, ThT fluorescence is a useful surrogate marker [28].

We observed that both valeric acid and butyric acid attenuated the conversion of $A \beta 1-40$ monomers to $A \beta 1$ 40 fibrils with a dose-response efficacy; the higher dose of valeric acid or butyric acid (SCFA:A $\beta$ molar ratio of $4: 1$ ) appeared more effective than the lower dose (SCFA:A $\beta$ molar ratio of $4: 1$ ) (Figure $2(a, b)$ ). We found that valeric acid also attenuated the conversion of $A \beta 1-42$ monomers to $A \beta 1-42$ fibrils with a dose-response efficacy; the higher dose of valeric acid (SCFA:A $\beta$ molar ratio of $4: 1$ ) appeared more effective than the lower dose (SCFA:A $\beta$ molar ratio of 4:1) (Figure 2(c)). Lastly, we observed that butyric acid treatment also displayed a tendency to reduce the formation of $A \beta 1-42$ fibrils, but there are no observable differences between the lower and higher dose (Figure 2(d)).

Collectively, our evidence demonstrates that valeric acid (and to a lesser extent, butyric acid) is capable of interfering with the conversion of monomeric $A \beta 1-40$ and $A \beta 1-42$ into $A \beta$ fibrils.

\subsection{Effects of valeric acid on the morphologies of the $A \beta$ assemblies}

Our evidence from Figures 1 and 2 demonstrating that, among the six SCFAs we tested, valeric acid most potently interferes with the aggregation of $A \beta$ peptides into higherordered assemblies. Based on this, we continued and used $E M$ to monitor for effects of valeric acid on the morphologies of the $A \beta 1-40$ and $A \beta 1-42$ assemblies. Consistent with our prior observations [25], the incubation of $A \beta 1-40$ (Figure 3, panel I) or $A \beta 1-42$ (Figure 3, panel III) in the presence of vehicle leads to the generation of classical non-branched fibrils with helicity features. $A \beta$ incubation in the presence of valeric acid, at a SCFA:A $\beta$ molar ratio of $4: 1$, almost completely inhibited fibril formation from $A \beta 1-40$ (Figure 3, panel II) or A 1-42 (Figure 3, panel IV).

\section{Discussion}

The assembly of $A \beta$ peptides into low-n oligomers requires initial protein-protein interactions among $A \beta$ peptides. Our study is specifically designed to assess if a specific test compound is capable of interfering with such proteinprotein interactions and therefore to disrupt the process $A \beta$ assembly into low $\mathrm{n}$ neurotoxic oligomers. We used independent in vitro assays to investigate six SCFAs that are derived from Gl microbiota metabolism of dietary fibers for their potential effects in modulating the assembly of $A \beta 1-40$ and $A \beta 1-42$ peptides to soluble, neurotoxic $A \beta$ aggregates that play roles in $A D$ pathogenesis. Results from our PICUP assays revealed that select SCFAs, particularly valeric acid, butyric acid, and propionic acid are capable of interfering with initial protein-protein interactions, 


\section{Aß1-40}
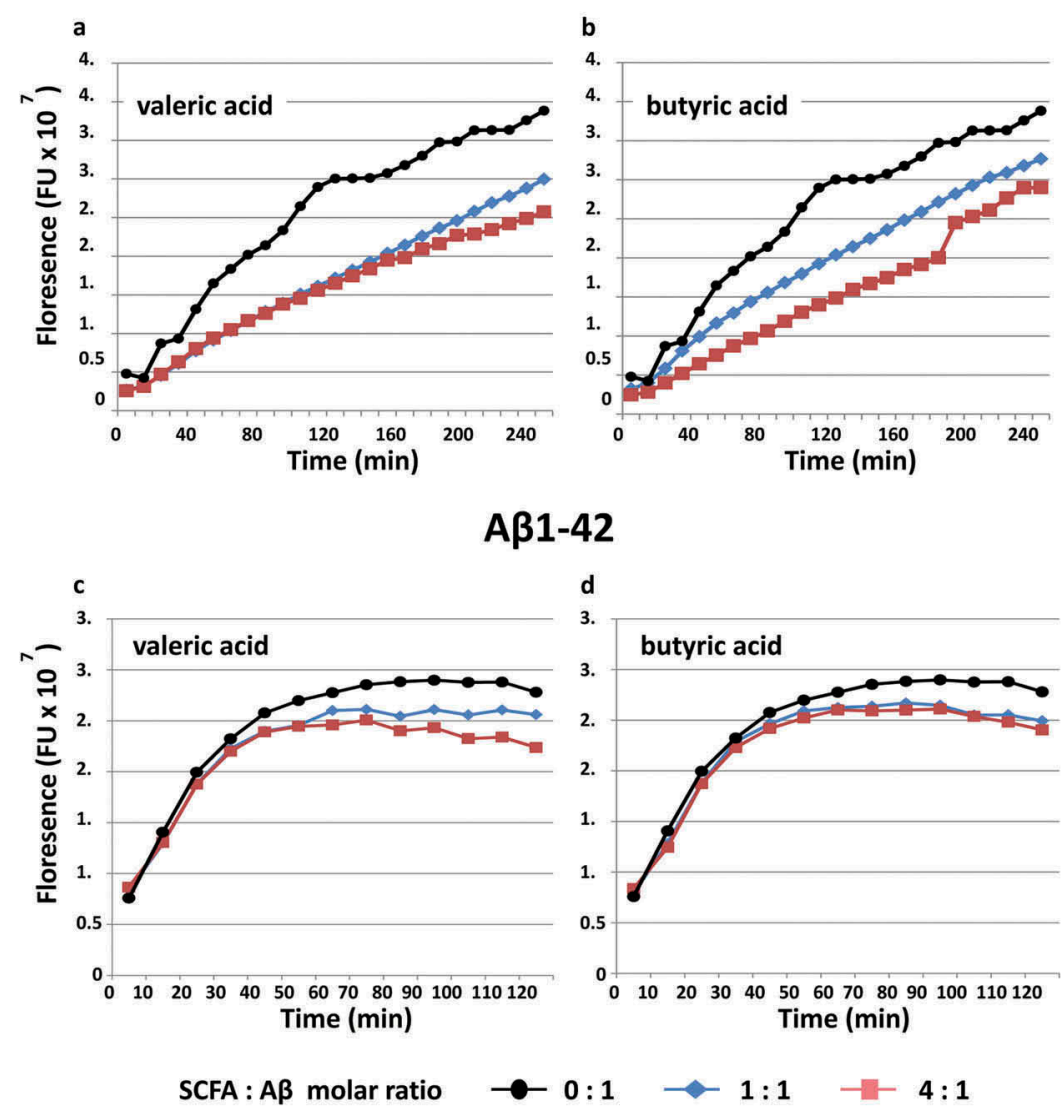

Figure 2. Select SCFAs potently interfere with A? fibril formation. Assembly of monomeric A?1-40 or A? 1-42 peptides into A? fibrils in the presence of valeric acid, butyric acid or vehicle were assessed using the ThT assay, which monitors ThT fluorescence as an indirect assessment of fibril contents. Periodically, aliquots were removed, and ThT binding levels were determined. Binding is expressed as mean fluorescence (in arbitrary fluorescence units (FU). (a-d) A?1-40 (a-b) or A?1-42 (c-d)

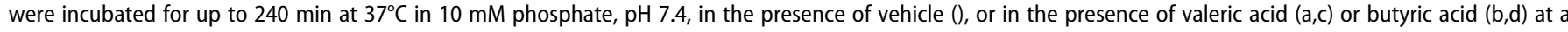
SCFA:A? molar ratio of 1:1 () or 4:1 (). Shown are representative assays from three independent studies.

which are necessary for $A \beta$ assemblies (Figure 1). In particular, valeric acid, butyric acid, and propionic acid demonstrated efficacy in interfering with protein-protein interactions that are necessary for the conversion of $A \beta$ peptides to neurotoxic $A \beta$ aggregates. The relative anti-A $\beta$ aggregation efficacy of the three SCFAs is, in decreasing order, valeric acid $\gg$ butyric acid $>$ propionic acid. In particular, valeric acid most potently inhibited protein-protein interaction of $A \beta 1-40$ and $A \beta 1-42$; the higher dose of valeric acid (at a SCFA:A $\beta$ molar ratio of 4:1) interfering with the aggregation of both $A \beta 1-40$ and 1-42 peptides. In comparison, the lower dose (at a valeric acid:A $\beta$ molar ratio of 1:1) was effective in interfering with the aggregation $A \beta 1-40$ into $A \beta 1-40$ trimers, and to a lesser extent $A \beta 1-40$ dimers, but was not effective in interfering with the aggregation of A 1 1-42. In contrast, butyric acid (at both the higher and lower dose) and propionic acid (at the higher dose) interfered with the formation of $A \beta 1-40$ trimers, but not the formation of $A \beta 1-40$ dimers. Moreover, either butyric acid or propionic acid was effective in modulating the aggregation of A $1-40$ peptides into higher-order aggregate forms. In contrast, three of the SCFAs tested (isobutyric acid, isovaleric acid, and acetic acid) had no observable effects on $A \beta$ assemblies, as assessed by the PICUP assay. The efficacy of valeric acid and butyric acid in interfering with the assemblies of higher-ordered $A \beta$ aggregate forms detectable by the PICUP assay is validated by observations from the independent ThT assay (Figure 2). Consistent with results from our PICUP assays, we observed that valeric acid and butyric acid were effective in interfering with the assembly of $A \beta 1-40$, and to a lesser extent, the assembly of $A \beta 1-42$ into $A \beta$ fibrils. The efficacy of valeric acid in interfering with the generation of $A \beta$ fibrils was validated by the EM assay, which demonstrated that valeric acid inhibited the assembly of $A \beta 1-40$ or $A \beta 1-42$ into $A \beta$ fibrils with classical non-branched fibrils with helicity features (Figure 3). Collectively, results from our studies support the hypothesis that intestinal microbiota may have protective effects against $A D$, in part, by supporting the generation of select SCFAs that interfere with the formation of toxic soluble $A \beta$ aggregates. While the microbiota is known for generating SCFAs for dietary fibers, we also note that relatively minor amount of SCFA can be of dietary nature. Thus it is possible that dietary SCFA may also 

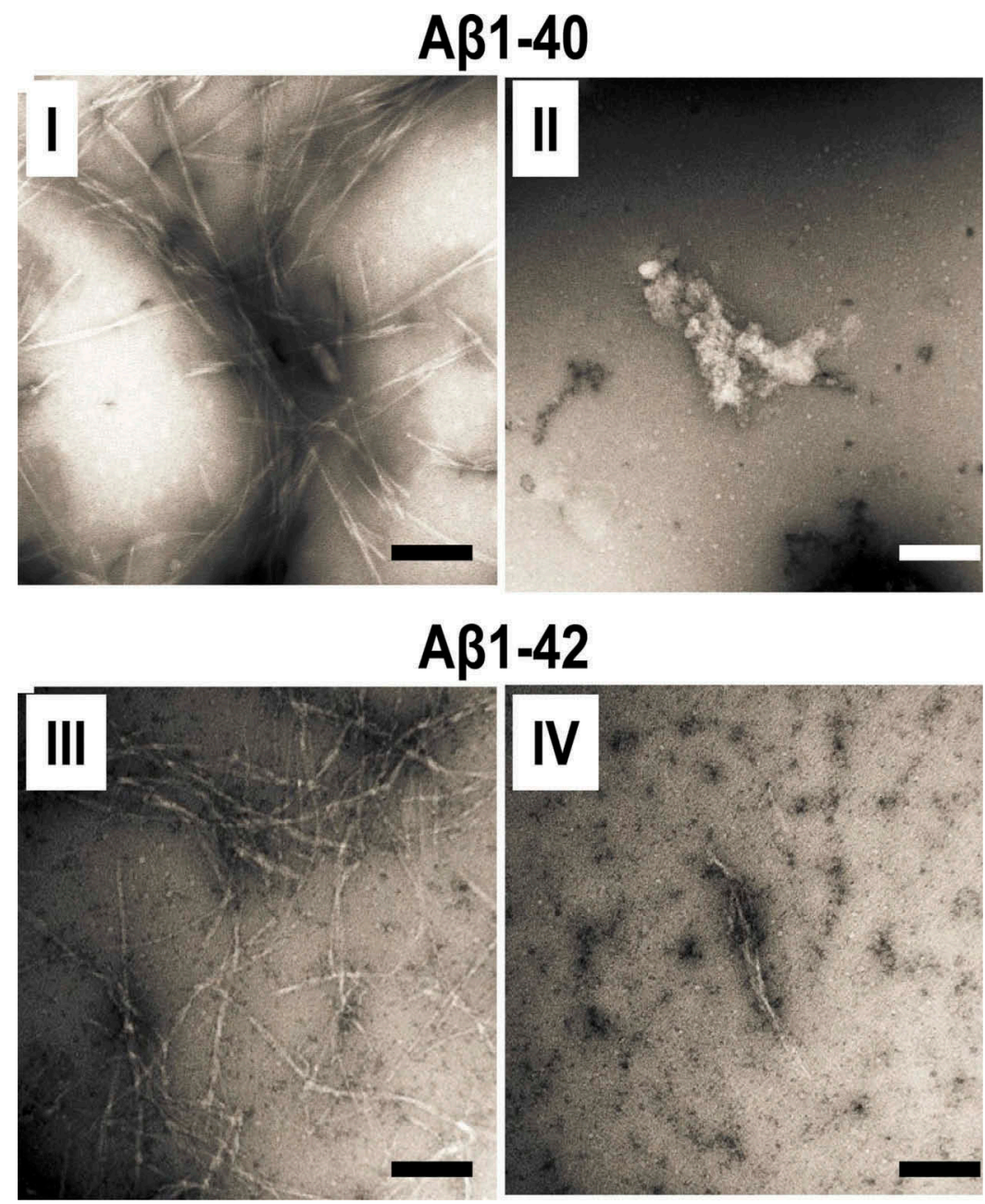

Figure 3. Assessments of $A$ ? protofibril morphology.

(Panels I, II, III, IV) EM was used to determine the morphologies of protofibrils obtained by the incubation of A?1-40 (panels I and II) or A?42 (panels III and IV), in the presence of vehicle (panels I and III) or valeric acid at a valeric acid:A? molar ratio of 4:1 (panels I and IV). Shown are representative assays from three independent studies. Scale bars indicate $100 \mathrm{~nm}$.

modulate $A \beta$ aggregation independent of contribution from Gl microbiota.

The misfolding of disease-specific proteins into toxic aggregates is an important and common pathological mechanism underlying diverse neurological disorders. Aside from misfolding of $A \beta$ peptides in $A D$, there are many other neurological disorders in which protein misfolding plays a crucial role in disease pathology, such as misfolding of a-synuclein in a-synucleinopathies such as PD, dementia with Lewy bodies, and multiple system atrophy, and misfolding of tau in tauopathies such as $A D$, dementia pugilistica, progressive supranuclear palsy, among others [29-32]. These aggregating proteins all share similar biophysical and biochemical properties that influence how they misfold, aggregate, and propagate in disease [33]. Thus, in addition to interfering with abnormal aggregation of amyloidogenic $A \beta$ isoforms, valeric acid, butyric acid, and propionic acid may also similarly interfere with the assembly of a-synuclein and tau.

Accumulating published evidence suggests that GI bacteria may help promote resilience against $A D$ through multiple mechanisms, the promotion of brain energy metabolism, modulation of neuro-inflammation, and modulation of epigenetic mechanisms (see Figure 4). Our evidence revealed that $\mathrm{Gl}$ bacteria may also improve $A D$ by supporting the generation of select SCFAs that are capable of interfering with the assembly of $A \beta 1-40$ and $A \beta 1-42$ peptides into neurotoxic $A \beta$ aggregates (see Figure 4). Future in vivo studies will be necessary to clarify whether certain SCFAs may attenuate AD $\beta$-amyloidosis through additional mechanisms.

\section{Conclusion}

Our observations provide the impetus for new investigations to identify and characterize select dietary fibers that support the generation of valeric acid, butyric acid, and propionic acid, as well as Gl microbial that are capable of metabolizing dietary fibers to the these SCFAs. Information gathered will lead to the development of next-generation probiotics that might help promote resilience to diverse neurodegenerative disorders. 


\section{GI Bacteria}

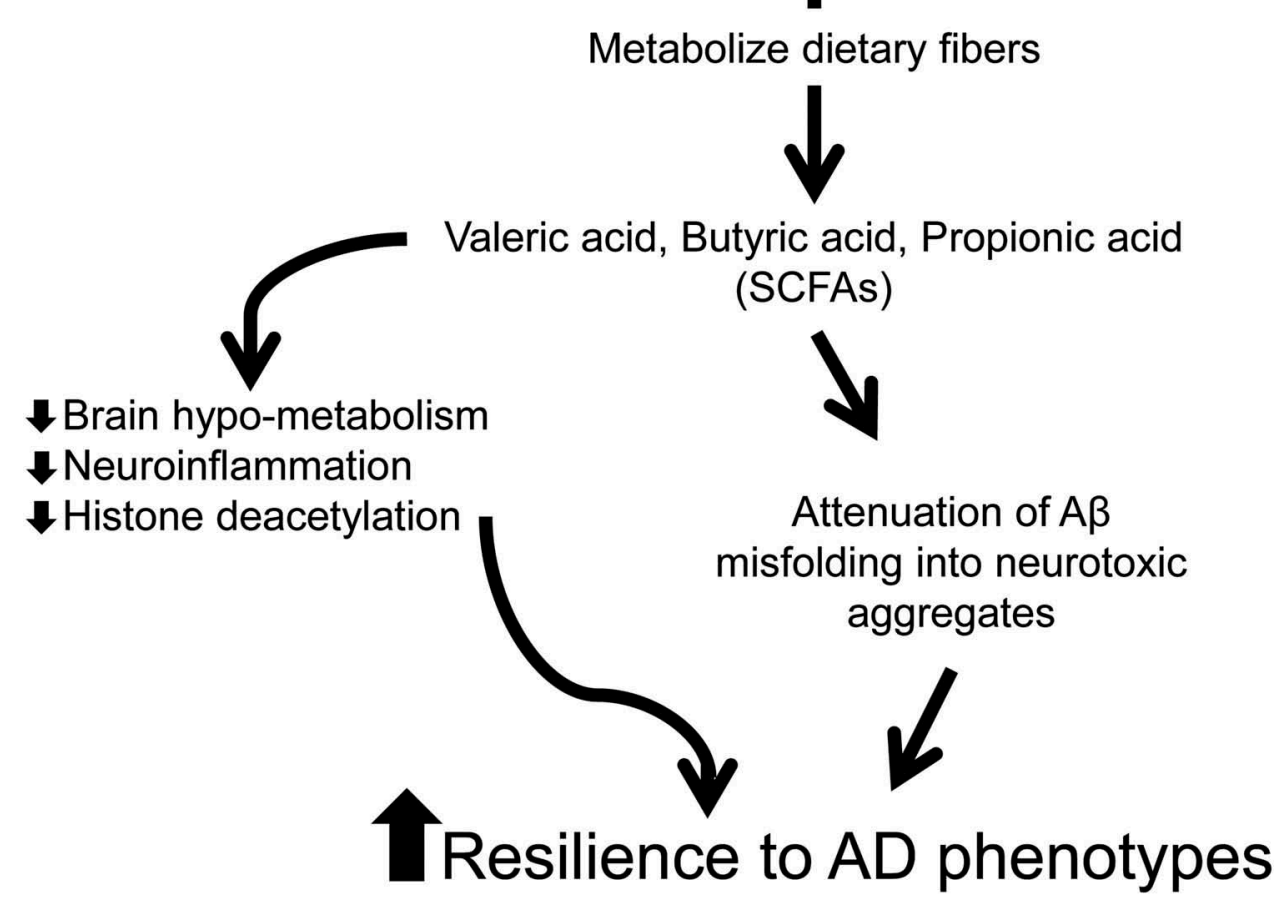

Figure 4. Schematics summarizing the mechanisms by which GI microbial-derived SCFAs may modulate AD.

Intestinal bacteria help protect against $A D$ by converting dietary fibers into biologically available SCFAs, which may promote resilience to $A D$ through multiple cellular/molecular mechanisms. Previously published evidence suggests SCFAs may benefit AD by: (1) alleviating brain hypo-metabolism as SCFAs provide alternative substrates for brain energy metabolism [20], (2) attenuating neuro-inflammation by modulating the maturation and function of microglia in the brain [5], and (3) inhibiting histone deacetylases and normalize aberrant histone acetylation in the $A D$ brain $[7,14,34]$. In addition, evidence from the present study suggests that certain SCFAs, particularly valeric acid, butyric acid, and propionic acid, may also benefit $A D$ by attenuating $A \beta$-mediated pathologic processes by interfering with the assembly of A?1-40 and A?1-42 peptides into neurotoxic A? aggregates.

\section{Key issues}

- The studies suggest that select bacterial species that support conversion of dietary fibers to short-chain fatty acids, which are relevant to neurodegenerative conditions.

- Our observation links gastrointestinal microbiota with mechanisms underlying $A D$-type $A \beta$ neuropathological mechanisms.

- Our observations suggest the feasibility of developing valeric acid for treating AD.

- Misfolding of diverse proteins in multiple neurological disorders, such as $A \beta$ in Alzheimer's disease, tau in tauopathies, a-synuclein in Parkinson's disease and Huntington's disease, all share common mechanistic features. Our observation supports the potential development of valeric acid for treating these diverse neurological disorders.

- Our observations support further investigations to identify and characterize gastrointestinal bacterial specie(s) that support the generation of valeric acid.

- Our observations also support further investigations to characterize select dietary fiber preparations that supports the generation of valeric acids.

- Information from the study will lead to the development of next-generation probiotics that might help promote resilience to diverse neurodegenerative disorders.

\section{Funding}

This study was supported in part by Grant Number P50 AT008661-01 from the NCCIH and the ODS. GM Pasinetti holds a VA Career Scientist Award. The authors acknowledge that the contents of this study do not represent the views of the NCCIH, the ODS, the NIH, the U.S. Department of Veterans Affairs, or the United States Government.

\section{Declaration of interest}

The authors have no relevant affiliations or financial involvement with any organization or entity with a financial interest in or financial conflict with the subject matter or materials discussed in the manuscript. This includes employment, consultancies, honoraria, stock ownership or options, expert testimony, grants or patents received or pending, or royalties.

\section{ORCID}

Paolo Mazzola (iD http://orcid.org/0000-0002-0484-1136

\section{References}

1. Li D, Wang $P$, Wang $P$, et al. The gut microbiota: a treasure for human health. Biotechnol Adv. 2016;34(7):1210-1224.

2. Scheperjans F, Aho V, Pereira PA, et al. Gut microbiota are related to Parkinson's disease and clinical phenotype. Mov Disord. 2015;30 (3):350-358. 
3. Sherwin E, Dinan TG, Cryan JF. Recent developments in understanding the role of the gut microbiota in brain health and disease. Ann N Y Acad Sci 2017;1406 [Epub ahead of print].

4. Wu SC, Cao ZS, Chang KM, et al. Intestinal microbial dysbiosis aggravates the progression of Alzheimer's disease in Drosophila. Nat Commun. 2017;8(1):24.

5. Sanchez B, Delgado S, Blanco-Miguez A, et al. Probiotics, gut microbiota, and their influence on host health and disease. Mol Nutr Food Res. 2017;61(1):1600240.

6. Koh A, De Vadder F, Kovatcheva-Datchary P, et al. From dietary fiber to host physiology: short-chain fatty acids as key bacterial metabolites. Cell. 2016;165(6):1332-1345.

7. Den Besten G, Van Eunen K, Groen AK, et al. The role of short-chain fatty acids in the interplay between diet, gut microbiota, and host energy metabolism. J Lipid Res. 2013;54(9):2325-2340.

8. Mori T, Koyama N, Guillot-Sestier MV, et al. Ferulic acid is a nutraceutical $\beta$-secretase modulator that improves behavioral impairment and Alzheimer-like pathology in transgenic mice. PLoS One. 2013;8(2):e5574.

9. Oboh G, Agunloye OM, Akinyemi AJ, et al. Comparative study on the inhibitory effect of caffeic and chlorogenic acids on key enzymes linked to Alzheimer's disease and some pro-oxidant induced oxidative stress in rats' brain-in vitro. Neurochem Res. 2013;38(2):413-419.

10. Wang Y, Wang Y, Li J, et al. Effects of caffeic acid on learning deficits in a model of Alzheimer's disease. Int J Mol Med. 2016;38 (8):869-875.

11. Clifford MN. Diet-derived phenols in plasma and tissues and their implications for health. Planta Med. 2004;70(12):1103-1114.

12. Monagas $M$, Uri-Sarda M, Sanchez-Patan F, et al. Insights into the metabolism and microbial biotransformation of dietary flavan-3-ols and the bioactivity of their metabolites. Food Funct. 2010;1(3):233-253.

13. Vetrani C, Rivellese AA, Anuzzi G, et al. Metabolic transformations of dietary polyphenols: comparison between in vitro colonic and hepatic models and in vivo urinary metabolites. J Nutr Biochem. 2016;33:111-118.

14. Menendez JA, Vazquez-Martin A, Oliveras-Ferraros C, et al. Analyzing effects of extra-virgin olive oil polyphenols on breast cancer-associated fatty acid synthase protein expression using reverse-phase protein microarrays. Int J Mol Med. 2008;22(4):433-439.

15. Xie L, Lee SG, Vance TM, et al. Bioavailability of anthocyanins and colonic polyphenol metabolites following consumption of aronia berry extract. Food Chem. 2016;211:860-868.

16. Sadeghi ES, Sleno L, Sabally K, et al. Biotransformation of polyphenols in a dynamic multistage gastrointestinal model. Food Chem. 2016;204:453-462.

17. Wang D, Ho L, Faith J, et al. Role of intestinal microbiota in the generation of polyphenol-derived phenolic acid mediated attenuation of Alzheimer's disease $\beta$-amyloid oligomerization. Mol Nutr Food Res. 2015;59(6):1025-1040.
18. Macfarlane GT, Bacteria MS. Colonic fermentation, and gastrointestinal health. J AOAC Int. 2012;95(1):50-60.

19. Cummings JH, Pomare EW, Branch WJ, et al. Short chain fatty acids in human large intestine, portal, hepatic and venous blood. Gut. 1987;28(10):1221-1227.

20. Zilberter $Y$, Zllberter $M$. The vicious circle of hypometabolism in neurodegenerative diseases: ways and mechanisms of metabolic correction. J Neurosci Res. 2017;95(11):2217-2235.

21. Erny D, Hrabe De Angelis AL, Jaitin $D$, et al. Host microbiota constantly control maturation and function of microglia in the CNS. Nat Neurosci. 2015;18:96-5977.

22. Martins IJ, Wmad BF. High fiber diets and Alzheimer's disease. Food and Nutr Sci. 2014;5(4):410-424.

23. Walsh DM, Lomakin A, Benedek GB, et al. Amyloid beta-protein fibrillogenesis. Detection of a protofibrillar intermediate. J Biol Chem. 1997;272(35):22364-22372.

24. Teplow DB. Preparation of amyloid beta-protein for structural and functional studies. Methods Enzymol. 2006;413:20-33.

25. Ono K, Condron MM, Ho L, et al. Effects of grape seed-derived polyphenols on amyloid beta-protein self-assembly and cytotoxicity. J Biol Chem. 2008;283(47):32176-32187.

26. Bitan G, Tarus B, Vollers SS, et al. A molecular switch in amyloid assembly: met35 and amyloid beta-protein oligomerization. J Am Chem Soc. 2003;125(50):15359-15365.

27. Bitan G, Fradinger EA, Spring SM, et al. Neurotoxic protein oligomers - what you see is not always what you get. Amyloid. 2015;12 (2):88-95.

28. LeVine $\mathrm{H}$. Quantification of beta-sheet amyloid fibril structures with thioflavin T. Methods Enzymol. 1999;309:274-284.

29. Kumar V, Sami N, Kashav T, et al. Protein aggregation and neurodegenerative diseases: from theory to therapy. Eur J Med Chem 2016;124:1105-1120.

30. Shamsi TN, Athar T, Parveen R, et al. A review on protein misfolding, aggregation and strategies to prevent related ailments. Int J Biol Macromol. 2017;105(Pt 1):993-1000.

31. Forloni G, Artuso V, La Vitola P, et al. Oligomeropathies and pathogenesis of Alzheimer and Parkinson's diseases. Mov Disord. 2016;31 (6): $771-781$

32. Pula JH, Kim J, Nichols J. Visual aspects of neurologic protein misfolding disorders. Curr Opin Ophthalmol. 2009;20(6):482489.

33. Ugalde CL, Finkelstein DI, Lawson VA, et al. Pathogenic mechanisms of prion protein, amyloid- $\beta$ and $\alpha$-synuclein misfolding: the prion concept and neurotoxicity of protein oligomers. J Neurochem. 2016;139(2):162-180.

34. Plagg B, Ehrlich D, Kniewallner KM, et al. Increased acetylation of histone $\mathrm{H} 4$ at lysine $12(\mathrm{H} 4 \mathrm{~K} 12)$ in monocytes of transgenic Alzheimer's mice and in human patients. Curr Alzheimer Res. 2015;12(8):752-760. 